\title{
Cloning of Feline cDNA Encoding the Cytotoxic T Lymphocyte-Associated Antigen 4 (CTLA-4)
}

\author{
Koichi OHNO, Miho FUJIKI, Tanvir S. KHATLANI, Hisashi INOKUMA and Takafumi ONISHI \\ Laboratory of Veterinary Internal Medicine. Faculty of Agriculture, Yamaguchi University, 1677-1 Yoshida, Yamaguchi 753-8515, \\ Japan \\ (Received 24 May 1999/Accepted 2 July 1999)
}

ABSTRACT. Cytotoxic T lymphocyte-associated antigen 4 (CTLA-4) is a CD28 homologue which down-modulate T cell responses rather than augment them. To investigate its biological role in feline immune system, we cloned and sequenced full-length feline CTLA-4 (fCTLA-4) cDNA by RT-PCR from pokeweed mitogen stimulated peripheral blood lymphocytes. The fCTLA-4 contains an open reading frame of 669 nucleotides, coding for a polypeptide of 223 amino acids. The predicted fCTLA- 4 amino acids sequence shows the homology of $86.6 \%, 87.0 \%$, and $76.2 \%$ with human, bovine, and murine molecules respectively. The hexapeptide motif (MYPPPY) within the extra-cellular domain of CTLA-4 molecule, which is believed to be responsible for interaction with the B7 family members, is completely conserved in all the species. —KEY WORDS: CD28, CTLA-4, feline.

J. Vet. Med. Sci. 61(11): 1241-1244, 1999

Activation of naive $\mathrm{T}$ cell requires the engagement of its antigen-specific $\mathrm{T}$ cell receptor (TCR) by a peptide antigen bound to a major histocompatibility complex (MHC) [1, 19]. A successful immune response, however, usually requires the engagement of additional "costimulatory" receptors $[16,17]$. One of these costimulatory signals occurs by the binding of CD28 on T cells to the CD80 (B7-1) and CD86 (B7-2) counter-receptors on antigen presenting cells [13]. Cytotoxic T lymphocyte-associated antigen 4 (CTLA$4 / \mathrm{CD} 152)$ is a surface glycoprotein that was initially identified through its involvement in cytotoxic $\mathrm{T}$ cell activation [2]. CTLA-4 shares some homology with CD28 and binds to the same ligands, B7 families [12]. Whereas CD28 is expressed on resting and activated T cells [4], CTLA-4 is found only on activated T-cells [12]. Initial activation of $\mathrm{T}$ cells after antigen exposure, is mediated by CD28/B7 interactions and leads to the proliferation and differentiation of effector $\mathrm{T}$ cells. Activated $\mathrm{T}$ cells express CTLA-4 on their surface which can bind the same B7 family ligands with higher affinity and downmodulate the $\mathrm{T}$ cell response rather than augment them [18]. The net cell response would then be a result of a balance between CD28 and CTLA-4 mediated events [7]. Thus CTLA-4 serves as a negative regulator of $\mathrm{T}$ cell activation [18]. CTLA-4 gene and cDNA has so far been cloned in many species including human [3], rabbit [6], cattle and chicken, [15], rat [14], and mouse [2] etc. We hereby report the cloning and sequencing of feline CTLA-4 to investigate the biological role of CTLA-4 in feline immune system.

Oligonucleotide primers that would amplify the full sequence of cDNA encoding feline CTLA-4 (fCTLA-4), were designed based on the 5' end and 3 ' end conserved regions of the human [3], mouse [2, 5] and rabbit [6] CTLA4 gene sequences. The $5^{\prime}$ primer sequence was 5'-GGTTTYGCTCTRCTTCCTGA-3' (where $\mathrm{Y}=\mathrm{C} / \mathrm{T}$ and $\mathrm{R}=\mathrm{A} / \mathrm{G}$ ) corresponding to mouse gene sequence (GenBank accession number X05719) [2] position number 11-30, and the $3^{\prime}$ end specific primer was 5'-AAAGTTYYAAT
TGCCTCAGCT-3' (where Y=T/C), corresponding to mouse gene sequence position numbers 773-793.

cDNA was constructed from total RNA obtained from the peripheral blood lymphocytes of a healthy cat that had been stimulated by pokeweed mitogen for $24 \mathrm{hr}$. Total RNA was isolated by using a RNeasy Mini Kit (QIAGEN, Hilden, Germany), according to the instructions of manufacturer. cDNA was constructed by using a oligonucleotide primer containing $17 \mathrm{dT}$ residues. This cDNA was amplified by polymerase chain reaction (PCR), by using the gene specific primers, and which resulted in a single band of about 780bp in length. This product was cloned in pGEM-T easy vector (Promega Corporation, Madison, WI, U.S.A.) and subjected to sequence analysis, on both sides by T7 and SP6, sequencing primers, by using Thermo Sequenase pre-mixed cycle sequencing kit (Amersham Pharmacia Biotech, Uppsala, Sweden). Three individually generated and sequenced clones showed no polymorphism. Nucleotide sequence and predicted amino acid sequence are shown in Fig. 1 (GenBank accesion number AF153202). fCTLA-4 has an open reading frame of 669 base pairs (bp), coding for a polypeptide of 223 amino acids (aa). Coding sequence starts from ATG at position 47 and terminates at the stop codon TGA at position 718 of the nucleotide (nt) sequence. When compared with the nt sequence of other species, it was found to be $87.7 \%$, $87.5 \%$, and $79.3 \%$, homologous to human, bovines, and murine CTLA-4 cDNA molecules respectively. Predicted aa sequence of fCTLA-4 molecule is shown in alignment with homologous molecules from other species in Fig. 2. When compared with the amino acid sequence of other species, it showed the homology of $86.6 \%, 87.0 \%$, and $76.2 \%$ with human, bovine, and murine molecules respectively. Based on the hydropathicity plot [10] and by comparison with already published sequence data of other species [15], the aa sequence of feline CTLA-4 is expected to have a hydrophobic leader sequence of 37 aa, followed by a extra-cellular domain of 124 aa, a transmembrane 
$\begin{array}{lllll}10 & 20 & 30 & 40 & 50\end{array}$ gottttgctctgcttcctgaagacctgaacactgctccataaagccatggctggctttgg $\begin{array}{llllll}M & A & G & F & G\end{array}$ $\begin{array}{lllll}70 & 80 & 90 & 100 & 110\end{array}$ attccggaggcatggggctcagccggacctggcttctaggacctggccctgcactgctct $\begin{array}{llllllllllllllllllll}F & R & R & H & G & A & Q & P & D & L & A & S & R & T & W & P & C & T & A & L\end{array}$ $\begin{array}{lllll}130 & 140 & 150 & 160 & 170\end{array}$ gtttctcttctcttatcccogtcttctccaaagggatgcatgtggctcagcctgcagt

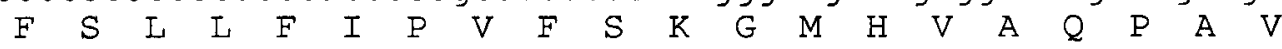
$\begin{array}{lllll}190 & 200 & 210 & 220 & 230\end{array}$ ggttctggccagcagccggggtgttgctagcttcgtgtgtgaatatgggtcttcaggcaa $\begin{array}{llllllllllllllllllll}V & L & A & S & S & R & G & V & A & S & F & V & C & E & Y & G & S & S & G & N\end{array}$ $\begin{array}{lllll}250 & 260 & 270 & 280 & 290\end{array}$ cgcagccgaggtccgggtgacagtgctgcggcaggctggcagccagatgactgaagtctg $\begin{array}{llllllllllllllllllll}A & A & E & V & R & V & T & V & L & R & Q & A & G & S & Q & M & T & E & V & C\end{array}$ $\begin{array}{lllll}310 & 320 & 330 & 340 & 350\end{array}$ tgccgtgacatacacagtggaggatgagttggccttcctggatgattctacctgcactgg $\begin{array}{llllllllllllllllllll}A & V & T & Y & T & V & E & D & E & I & A & F & I & D & D & S & T & C & T & G\end{array}$ $\begin{array}{lllll}370 & 380 & 390 & 400 & 410\end{array}$ cacctccagtggaaacaaagtgaacctcaccatccaagggttgagggccatggacacggg

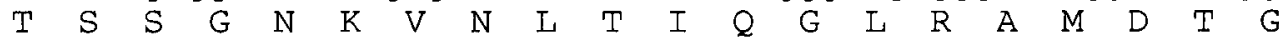
$430 \quad 440 \quad 450 \quad 460 \quad 470 \quad 480$ gctctacatctgcaaggtggagctcatgtacccaccaccctactatgtaggcatgggaaa $\begin{array}{llllllllllllllllllll}I & Y & I & C & K & V & E & I & M & Y & P & P & P & Y & Y & V & G & M & G & N\end{array}$

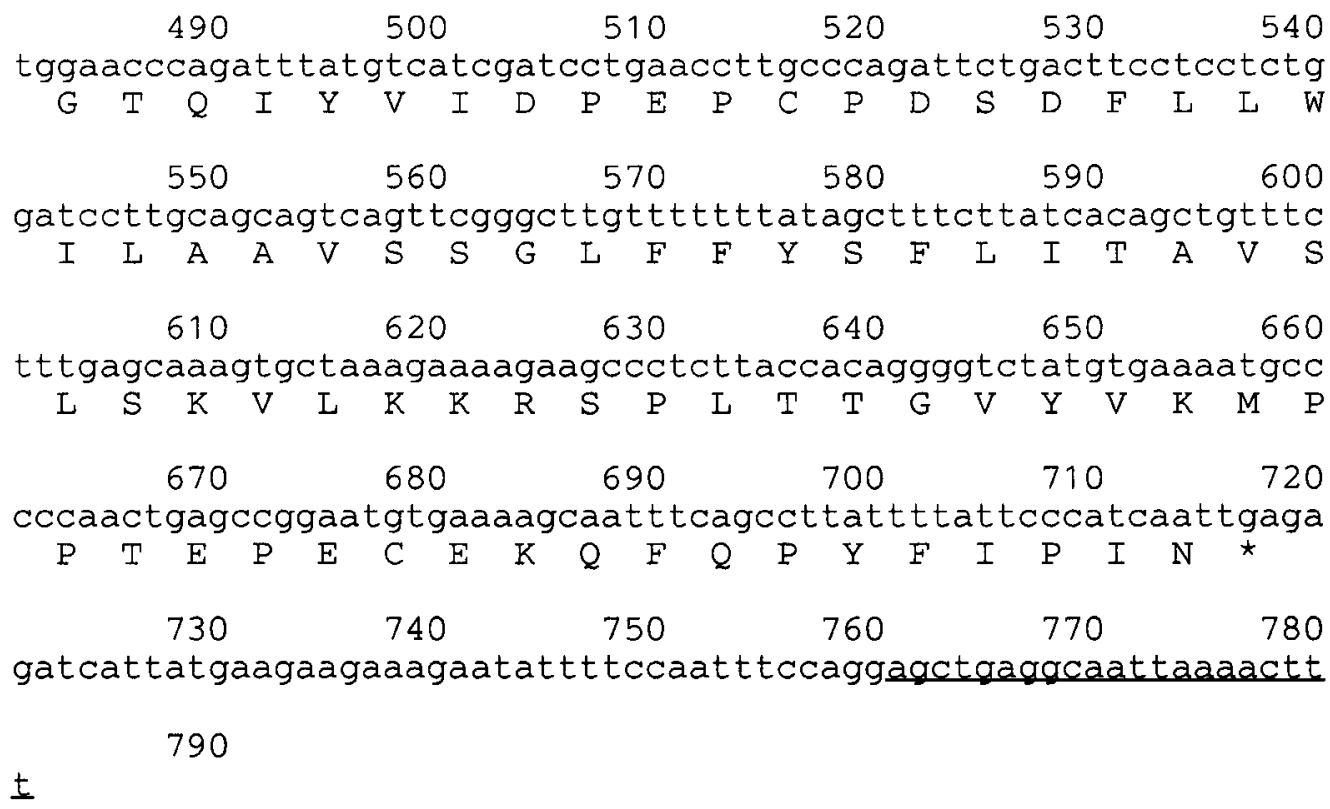

Fig. 1. Nucleotide and deduced amino acid sequence of feline CTLA-4 molecule (GenBank accesion number AF153202). The gene specific primers used to amplify feline CTLA-4 gene are underlined.

region of 23 aa, and a cytoplasmic tail of 39 aa. The hexapeptide motif (MYPPPY) within the extra-cellular domain of CTLA-4 molecule is completely conserved in all the species. As this motif is believed to be responsible for 


\section{$\longrightarrow \quad(L$ e a d er $\quad$ Seque $\mathrm{n}$ ce)}

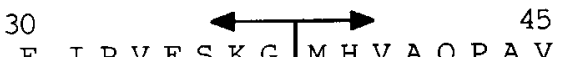
Fe. MAGFGFRR H A P D L A S T T P C T A L F S L L F I P V F S G M H A Q P A V

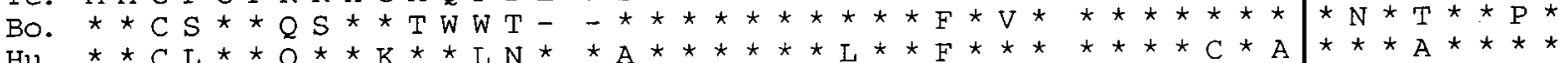

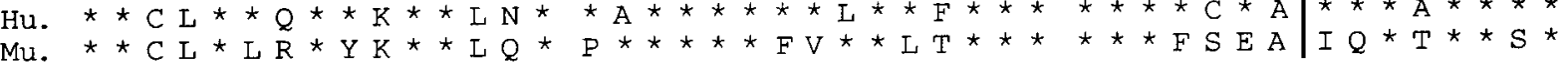

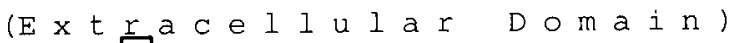

Fe. VLASSRGVASFVDEY GSSGNAAEVRVTVLR QAGSQMTEVCAVTYT

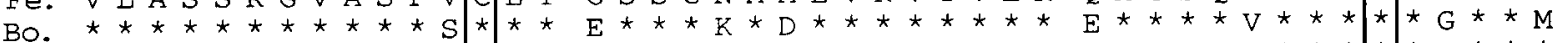

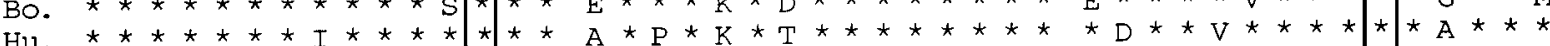

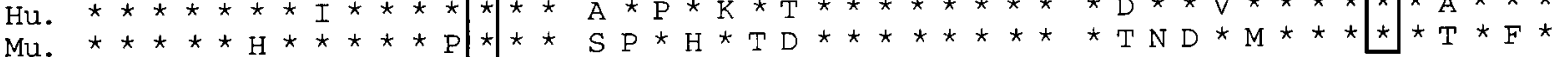

\section{5} 120

Fe. VEDELAFLDDSTCTG TSSGNKVNLTIQGLRAMDTGLYICKVELMY

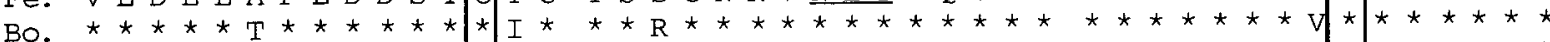

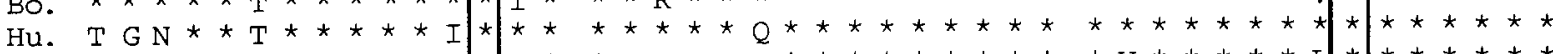

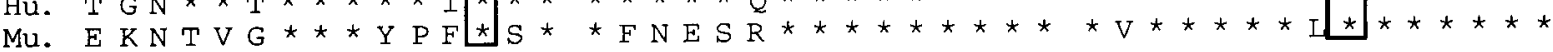

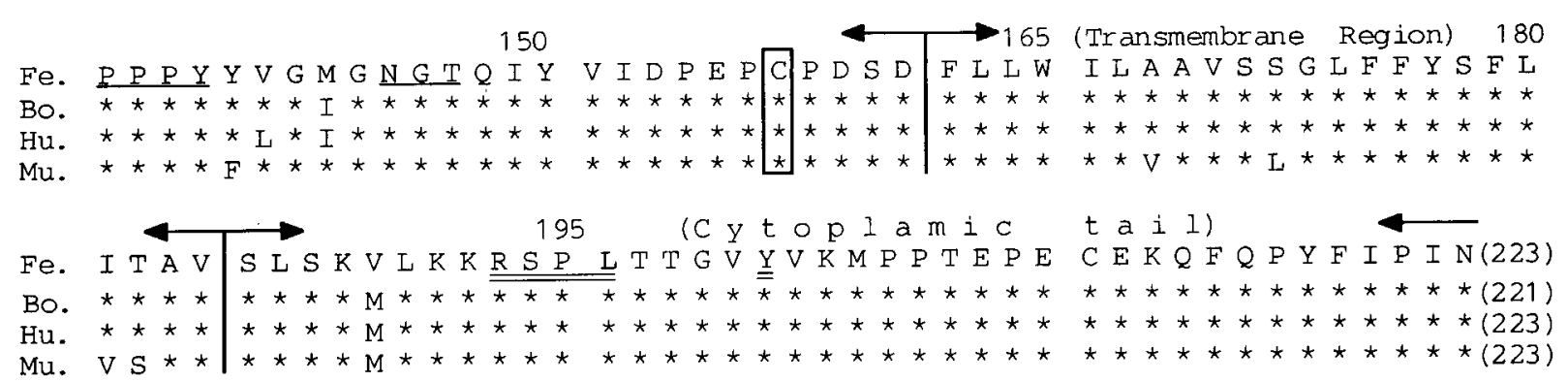

Fig. 2. Alignment of predicted feline (Fe) CTLA-4 amino acid sequence with their homologues from different species, bovines (Bo), human $(\mathrm{Hu})$ and mouse (Mo). Expected leader sequence, extra-cellular domain, transmembrane region, cytoplasmic tail, and conserved cysteine residues (closed boxes) in the extra-cellular region are shown. Dashes (-) are introduced where sequence fails. MYPPPY hexapeptide motif and potential glycosylation sites are underlined. Two potential sites for phosphorylation in cytoplasmic tail, RSPL with a lysine residue nearby and a tyrosine residue adjacent to the RSPL motif, are double-underlined. The amino acids residues are aligned to get maximum homology. Numerals at the end show the total number of amino acid residues.

interaction with the B7 family members, its conservation among these species may explain the species specificity. There are two predicted N-linked glycosylation sites in the sequence, which are conserved in all the species. There are 5 cysteine residues in the $\mathrm{V}$ like extra cellular domain and all of them show complete identity with other species. The conserved cytoplasmic tail of CTLA- 4 contains two potential sites for phosphorylation [9], first the sequence XSPX (RSPL, with a lysine residue nearby) which could be recognized by a proline-dependant protein kinase, and second, a tyrosine residue adjacent to the RSPL motif. The cytoplasmic tail shows the high homology with other species (Fig. 2), thus establishing a trend for conservation of these motifs for signal transduction.

It is concluded that we have cloned and sequenced the cDNA encoding the full-length fCTLA-4 molecule, which exhibits high homology to human and cattle CTLA-4 at both the nucleic acid as well as the amino acid level. CTLA4 is an important regulator of the immune response, exerting its influence on reactivity to both foreign and self antigens. CTLA-4 blockade enhances T cell responses [8], augments antitumor immunity [11], and exacerbates an induced autoimmune diseases [7]. This study will provide a useful reagent, by examining its expression patterns, production of recombinant protein and raising antibodies against it, for finding its role in feline immune system and immunological diseases.

\section{REFERENCES}

1. Allen, P.M. 1987. Immunol. Today 8: 270.

2. Brunet, J.F., Denzoit, F., Luciani, M.F., Dosseto, M.R., Suzan, M., Mattei, M.G. and Golstein, P. 1987. Nature (Lond.) 328: 267-270

3. Dariavach, P., Mattei, M.G., Golstein, P. and Lefranc, M.P. 1988. Eur. J. Immunol. 18: 1901.

4. Hansen, J.A., Martin, P.J. and Nowinski, R.C. 1980. Immunogenetics 10: 247-260.

5. Harper, K., Balzano, C., Rouvier, E., Mattei, M.G., Luciani, M.F. and Golstein, P. 1991. J. Immunol. 147: 1037-1044.

6. Isono, T. and Seto, A. 1995. Immunogenetics 42: 217-220.

7. Karandikar, N.J., Vanderlugt, C.L., Walunas, T.L., Miller, S.D. and Bluestone, J.A. 1996. J. Exp. Med. 184: 783-788.

8. Kearney, E.R., Walunas, T.L., Karr, R.W., Morton, P.A., Loh,, D.Y., Bluestone, J.A. and Jenkins, M.K. 1995. J. Immunol. 155: $1032-1036$

9. Kemp, B.E. and Pearson, R.B. 1990. TIBS 15: 342-346.

10. Kyte, J. and Doolittle, R.F. 1982. J. Mol. Biol. 157: 105-132.

11. Leach, D.R., Krummel, M.F. and Allison, J.P. 1996. Science 271: 1734-1736.

12. Linsley, P.S., Brady, W., Urnes, M., Grosmaire, L.S., Damle, N.K. and Ledbetter, J.A. 1991. J. Exp. Med. 174: 561-569.

13. Linsley, P.S., Greene, J.L., Tan, P., Bradshaw, J., Ledbetter, J.A., Anaseti, C. and Damle, N. 1992. J. Exp. Med. 176: 
1595-1604.

14. Oaks, M.K., Penwell, R.T. and Tector, A.J. 1996. Immunogenetics 43: 173-174.

15. Parsons, K.R., Young, J.R., Collins, B.A. and Horward, C.J. 1996. Immunogenetics 43: 388-391.

16. Schwartz, R.H. 1990. Science 248: 1349-1356.
17. Schwartz, R.H. 1992. Cell 71: 1065-1068.

18. Walunas, T.L., Lenschow, D.J., Bakker, C.Y., Linsley, P.S., Freeman, G.J., Green, J.M., Thompson, C.B. and Bluestone, J.A. 1994. Immunity 1: 405-414.

19. Weiss, A. 1990. J. Clin. Invest. 86: 1015. 